\title{
Chern-Simons Gravities (CSG) and Gravitational Chern-Simons (GCS) densities in all dimensions
}

\author{
D. H. Tchrakian ${ }^{\star \dagger \ddagger}$ \\ ${ }^{\dagger}$ School of Theoretical Physics, Dublin Institute for Advanced Studies, 10 Burlington Road, Dublin 4, Ireland \\ ${ }^{\dagger}$ Department of Computer Science, Maynooth University, Maynooth, Ireland

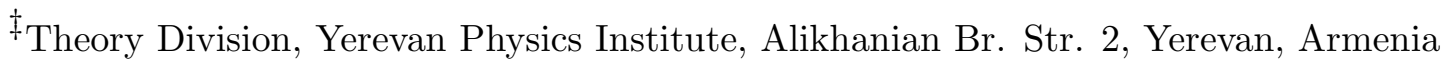

\begin{abstract}
Chern-Simons gravities and gravitational Chern-Simons densities are constructed using the non-Abelian Yang-Mills Chern-Simons densities. As such, they are defined only in odd dimensions. We propose instead an analogous construction employing what we term Higgs-Chern-Simons (HCS) densities, which are defined in all dimensions. This enables the definition of extended versions of Chern-Simons gravities in all dimensions. Employing the same prescription, the definition of gravitational Chern-Simons densities is extended to all even dimensions, but only to $4 p-1$ odd dimensions. All our considerations are restricted to vacuum fields.
\end{abstract}

Talk given at XVII International Conference on Symmetry Methods in Physics (Symphys XVII), Yerevan, Armenia, 09-15 July, 2017.

\section{Introduction}

In the context of this report, Chern-Simons gravities (CSG) and gravitational Chern-Simons (GCS) densities are distinct but not unrelated objects. They both result from the definition of Chern-Simons (CS) densities of the non-Abelian (nA) Yang-Mills (YM) fields. The CSG consist of some superpositioxn of usual gravitational Lagrangians displaying all orders of the Riemann curvature, including the 0 -th - the cosmological constant. The GCS are the direct gravitational analogues of the nA CS densities, and like these, they can be employed in conjuction with usual gravitational Lagrangians. 
CS gravity (CSG) in 2+1 dimensions was proposed in [1] and was subsequently extended to $2 n+1$ dimensions in [2, 3]. Gravitational CS densities in $2+1$ dimensions first appeared 1 ] in [4, 6] and were extended to odd dimensions in [7, 8].

Since the construction of both CSG's and GCS densities employ the nA CS densities, it follows that these are defined in odd dimensions only. The main aim here to extend these definitions to cover also even dimensions. Indeed, such examples are present in the literature both for CSG [3, 9], and, for GCS density [10, 5] in 3+1 dimensions.

For CSG systems in 3+1 dimensions, two such proposals are made, in [9] and in [3]. In the second of these, in [3], a Higgs-like 2 scalar is employed, which is contained in what is proposed in the present report. A GCS densities in 3+1 dimensions are introduced in [10] and [5], which are employed for the purpose of modifying the usual (Einstein) gravity. In [10, the (gravitational) Pontryagin density is employed, while in [5] both the Pontryagin and the Euler densities are employed. Definitions of GCS densities are made in this spirit in what follows here.

The pivotal point in our considerations is the explotation of (what is referred to here) HiggsChern-Simons (HCS) densities, which are defined in all, odd and even dimensions. These result from the dimensional descent of Chern-Pontryagin (CP) densities from some even dimension down to any even or odd dimension. They are introduced in [12, 13] and in Appendix A of [14. These HCS densities will be employed instead of the usual CS densities, to construct gravitational models in all dimensions which might be described as Higgs-Chern-Simons gravities (HCSG), and also to construct gravitational Higgs-Chern-Simons densities (GHCS) in all even dimensions, and in $4 p-1$ odd dimensions. It is known that GCS densities are absent [7, 8] in $4 p-3$ dimensions, and it will turn out that also GHCS densities are absent in $4 p-1$ dimensions.

All statements made are illustrated via typical exmples, and all calculations are carried out in the Einstein-Cartan formulation of gravity. In the Einstein-Cartan formulation, the gravitational system can be described in terms of (a) the Vielbein fields $e_{M}^{a}$ (and its inverse $e_{a}^{M}$ ) which are related to the metric through

$$
g_{M N}=e_{M}^{a} e_{N}^{b} \eta_{a b}, \quad g^{M N}=e_{a}^{M} e_{b}^{N} \eta^{a b},
$$

$\eta_{a b}$ being the flat (Minkowskian or Euclidean) metric, and (b) the spin-connection $\omega_{M}^{a b}$ which defines the Riemann curvature

$$
R_{M N}=\partial_{[M} \omega_{N]}^{a b}+\left(\omega_{[M} \omega_{N]}\right)^{a b} .
$$

Since the frame indices $a, b, \ldots$ are raised/lowered by a flat metric, and since we are not particularly concerned with the signature of the spaces(s), we will henceforth not pay any attention to whether the frame index is covariant or contravariant.

It is the identification of the spin-connection and the Riemann curvature with the YM connection and curvature in $D$ dimensions, through

$$
A_{M}=-\frac{1}{2} \omega_{M}^{a b} L_{a b} \Rightarrow F_{M N}=-\frac{1}{2} R_{M N}^{a b} L_{a b}, \quad M=1,2, \ldots, D, \quad a=1,2, \ldots, D
$$

\footnotetext{
${ }^{1}$ In these references the CS density is used to generate mass in a (Topological) non-Abelian field theory, and in the gravitational case the GCS density is added to the usual gravity.

${ }^{2}$ The scalar used in [3, and in the rest of this presentation, is not a matter field. We refer to it as Higgs because of its provenance via the dimensional descent of a non-Abelian (nA) density. The gravitational degrees of freedom it yields is technically related to the nA Higgs field as the spin-connection is related to the nA curvature.
} 
that is exploited in the definitions of both GCS densities and CSG gravities. The matrices $L_{a b}$ in (11) are representations of $S O(D)$. In what follows, we will employ the Dirac (Clifford algebraic) representations for $L_{a b}$

$$
L_{a b}=\gamma_{a b}=-\frac{1}{4}\left[\gamma_{a}, \gamma_{b}\right]
$$

in terms of $\gamma_{a}$, the gamma matrices in $D$ dimensions.

The Chern-Simons (CS) density expressed in terms of the YM connection $A_{M}$ and curvature $F_{M N}$ is defined through the one-step descent of the Chern-Pontryagin (CP) density

$$
\Omega_{\mathrm{CP}}=\operatorname{Tr} F \wedge F \wedge \cdots \wedge F=\nabla \cdot \Omega, \quad 2 n \text { times }
$$

in some even, $D=2 n$ dimensions [11]. The one-step descent in question is a result of the fact that the CP density $\Omega_{\mathrm{CP}}=\boldsymbol{\nabla} \cdot \boldsymbol{\Omega}$ is a total divergence. The CS density is defined as any one component of the vector-valued density $\Omega$, say $\Omega_{D}$, i.e. $\Omega_{\mathrm{CS}}=\Omega_{D}$, which now depends only on the $D-1$ coordinates $x_{\mu}, \quad \mu=1,2, \ldots, D-1$. Hence, the CS density thus defined necessarily exists in some odd dimension $2 n-1$. The CS densities are by construction gauge variant but their variational equations turn out to be gauge covariant, and display other interesting features prominent among which is their gauge transformation properties in the case of nA fields, leading to their exploitation in quantum field theory [4, 6]. These aspects of CS theory will not be pursued here. Instead, our aim here is to exploit the non-Abelian (nA) CS densities to construct gravitational-CS (GCS) densities and Chern-Simons gravities (CSG).

Already at this stage it is clear that the passage from YM to gravity prescribed by (11) is problematic in the context of gravitational CS (GCS) densities and CS gravities (CSG), since these are defined in $D-1$ dimensions with coordinates $x_{\mu}, \quad \mu=1,2, \ldots, D-1$, while the frame indices run over $a=1,2, \ldots, D$ instead of $D-1$. This discrepancy is corrected in each case respectively, GCS and CSG, by sharpening the prescription (11).

Following this prescription, it is clear that GCS densities and CSG systems can be defined only in odd dimensions. Our aim in this presentation is to propose GCS densities and CSG systems in all dimensions, namely in both odd and even dimensions. To this end, one starts from a version of the Chern-Pontryagin (CP) densities that is defined in odd and even dimensions. These are the Higgs-CP (HCP) densities resulting from the dimensional descent of a $\mathrm{CP}$ density in some even dimension, down to some odd or even residual dimension. The Higgs field in this case is a relic of the YM connection in the higher dimension and these HCP densities are total divergences like the CP densities.

The dimensional reduction of gauge fields has a long history [15, 16, 17]. The calculus of dimensional reduction used in [12, 13, 14] is an extended version of that of Ref. [16]. In most applications of this calculus, the descent was carried out on the Yang-Mills action/energy density in higher dimensons. Application to the descent of Chern-Pontryagin densities was first carried out in [18] applied to the third and fourth CP denisties in 6 and 8 dimensions down to 3 dimensions, yielding the monopole (topological) charge densities of two extended Yang-Mills-Higgs (YMH) theory on $\mathbb{R}^{3}$. Soon after in [19], the fourth $\mathrm{CP}$ denisty in 8 dimensions was dimensionally reduced down to 4 dimensions, yielding the monopole 3 (topological) charge density of a YMH theory on

\footnotetext{
${ }^{3}$ In [19], the descent was performed both on the 4-th CP density and the $p=2 \mathrm{YM}[20$ system to yield a YMH theory supporting "instantonss" on $\mathbb{R}^{4}$.
} 
$\mathbb{R}^{4}$. Subsequently this formulation was extended to all even and odd dimensions in Refs. [12, 13 and in Appendix A of Ref [14].

The total divergence property of the HCP densities enables, via the standard one-step descent, the definition of CS densities in all dimensions. We refer to these as Higgs-CS (HCS) densities resulting from the one-step descent - from $D$ to $D-1$ dimensions. In $3+1$ dimensions in particular, two such HCS densitied were employed in Refs. [21, 22] in $S O(5)$ and $S U(3)$ YMH models.

In arbitrary dimensions, the HCS densities are introduced in [12, 13, 14]. Recently, some HCS densities were given independently in [23]. While in many cases the HCS densities of [23] agree with ours [12, 13, 14], they differ most markedly in that they are defined in odd dimensions only, while in our case all, even and odd, dimensions are included. The reason for this is that in [23] it is the CS density 4 in the (higher) odd dimensions which is subjected to dimensional reduction, and then by 2 dimensions or or by some other even dimension. Thus in the case of [23], only even dimensional HCS are defined. In our case by contrast, it is the gauge invariant CP density that is subjected to dimensional reduction by any number of dimensions, resulting in HCS densities in odd dimensions. It turns out that in even residual dimensions, the HCS density thus obtained is gauge invariant like the CP density in the bulk.

It is the HCS densities that are employed in constructing the gravitational HCS (GHCS) densities and the HCS gravities (HCSG) in both odd and even dimensions, applying some variants of the prescription (1). It turns out that the construction of HCSG systems is unique, in the sense that in odd dimensions where CSG systems exist already before the introduction of the Higgs field, this CSG system is embedded in the corresponding HCSG system. The situation with the construction of the GCSH density is rather more ad hoc, such that in odd dimensions where both GCS and GHCS densities can be constructed, the two results are different. We have thus relegated the subject of GCS and GHCS to an Appendix.

The presentation is organised as follows. In Section 2 the main building blocks, namely the CS densities for $d=3,5,7$ and HCS densities for for $d=3,4,5,6.7$, dimensions $d=D-1$ are listed. In Section 3 some CS gravities (CSG) and HCS gravities (HCSG) are presented. In Section 4 some gravitational CS desities (GCS) and gravitational HCS (GHCS) densities are presented. The reason for the chosen order of Sections $\mathbf{3}$ and $\mathbf{4}$ is that in the context adopted here, the GCS (and GHCS) densities are objects which should be employed to modify the more fundamental CSG (and HCSG) systems.

\section{Chern-Simons and Higgs-Chern-Simons densities}

In this Section we present the usual Chern-Simons (CS) densities, which are defined in odd dimensions only, and the Higgs-Chern-Simons densities which are defined in all dimensions. They are defined for arbitrary gauge group but the choice of gauge group appropriate for the passage of Yang-Mills to gravity, is specified.

\footnotetext{
${ }^{4}$ Dimensional reduction being a calculus of symmetry imposition, it is unsafe to carry it out on a gauge variant density. That in some examples this may not be problematic [24, happens to be true.
} 


\subsection{The usual Chern-Simons densities in odd dimensions}

The usual Chern-Simons (CS) density in $d$ dimensions results from the one-step descent of the the Chern-Pontryagin (CP) density in $D=d+1$ dimensions, $D=2 n$ being even. Since our final aim is to transition from Yang-Mills to gravity, the gauge group of the non-Abelian field, is fixed by the prescription (11) where $L_{a b}$ takes its values in the algebra of $S O(D)(D=2 n)$ with $a=1,2, \ldots, 2 n$. The corresp[onding gravitational density is constructed by evaluating the trace in the CS formula. Since $D$ is even, $L_{a b}$ are represented the Dirac matrices $\gamma_{a}$, where these are augmented by the chiral matrix $\gamma_{D+1}$. One has then the option of including $\gamma_{D+1}$ in the trace of the nA CS density.

For the sake of illustration, we state the CS densities in $d=3,5,7$. Including $\gamma_{D+1}$ in the trace these are

$$
\begin{aligned}
\Omega_{\mathrm{CS}}^{(3)}= & \varepsilon^{\lambda \mu \nu} \operatorname{Tr} \gamma_{5} A_{\lambda}\left[F_{\mu \nu}-\frac{2}{3} A_{\mu} A_{\nu}\right] \\
\Omega_{\mathrm{CS}}^{(5)}= & \varepsilon^{\lambda \mu \nu \rho \sigma} \operatorname{Tr} \gamma_{7} A_{\lambda}\left[F_{\mu \nu} F_{\rho \sigma}-F_{\mu \nu} A_{\rho} A_{\sigma}+\frac{2}{5} A_{\mu} A_{\nu} A_{\rho} A_{\sigma}\right] \\
\Omega_{\mathrm{CS}}^{(7)}= & \varepsilon^{\lambda \mu \nu \rho \tau \kappa} \operatorname{Tr} \gamma_{9} A_{\lambda}\left[F_{\mu \nu} F_{\rho \sigma} F_{\tau \kappa}-\frac{4}{5} F_{\mu \nu} F_{\rho \sigma} A_{\tau} A_{\kappa}-\frac{2}{5} F_{\mu \nu} A_{\rho} A_{\sigma} F_{\tau \kappa}\right. \\
& \left.+\frac{4}{5} F_{\mu \nu} A_{\rho} A_{\sigma} A_{\tau} A_{\kappa}-\frac{8}{35} A_{\mu} A_{\nu} A_{\rho} A_{\sigma} A_{\tau} A_{\kappa}\right],
\end{aligned}
$$

This choice is appropriate for the construction of CS gravities [1, 2, 3] (CSG). It results in Euler type gravitational densities 25$]$.

The other choice is to exclude $\gamma_{D+1}$ in the trace. The resulting CS densities

$$
\begin{aligned}
& \hat{\Omega}_{\mathrm{CS}}^{(3)}=\varepsilon^{\lambda \mu \nu} \operatorname{Tr} A_{\lambda}\left[F_{\mu \nu}-\frac{2}{3} A_{\mu} A_{\nu}\right] \\
& \hat{\Omega}_{\mathrm{CS}}^{(5)}=\varepsilon^{\lambda \mu \nu \rho \sigma} \operatorname{Tr} A_{\lambda}\left[F_{\mu \nu} F_{\rho \sigma}-F_{\mu \nu} A_{\rho} A_{\sigma}+\frac{2}{5} A_{\mu} A_{\nu} A_{\rho} A_{\sigma}\right] \\
& \hat{\Omega}_{\mathrm{CS}}^{(7)=} \varepsilon^{\lambda \mu \nu \rho \sigma \tau \kappa} \operatorname{Tr} A_{\lambda}\left[F_{\mu \nu} F_{\rho \sigma} F_{\tau \kappa}-\right. \frac{4}{5} F_{\mu \nu} F_{\rho \sigma} A_{\tau} A_{\kappa}-\frac{2}{5} F_{\mu \nu} A_{\rho} A_{\sigma} F_{\tau \kappa} \\
&\left.+\frac{4}{5} F_{\mu \nu} A_{\rho} A_{\sigma} A_{\tau} A_{\kappa}-\frac{8}{35} A_{\mu} A_{\nu} A_{\rho} A_{\sigma} A_{\tau} A_{\kappa}\right],
\end{aligned}
$$

which are appropriate for the construction of gravitational CS densities, and result in Pontryagin type gravitational densities [25]..

\subsection{The Higgs Chern-Simons densities in all dimensions}

The definitions of the Higgs-Chern-Simons (HCS) densities however are more ubiquitous. Thus for example a HCS density in $d$ dimensions, which is derived from the Higgs-Chern-Pontryagin (HCP) density in $D=d+1, D$ being even or odd. The HCP density in question, itself arises from the dimensional descents of the $\mathrm{CP}$ in some even dimension $N \geq d+1$. 
The HCP density in $D$ density employed, say $\Omega_{\mathrm{HCP}}^{D, N}$, is descended from a CP density $\Omega_{\mathrm{CP}}$ in even $N$ dimensions. It may thus be useful to denote the HCS density gotten via the one-step descent as

$$
\Omega_{\mathrm{HCS}}^{(d, N)}, \quad d=D-1 .
$$

A detailed description of HCP and HCS description is given in [12, 14].

In the notation used below, the (suare matrix valued) Higgs scalar $\Phi$ has dimension $L^{-1}$, as does also the constant $\eta$ which is the inverse of the sphere over which the descent is carried out.

We list two such the HCS densities in $d=3$, arrived at from the one-step descents from the HCP densities in $D=4$, each of them descended from the CP densities in 6 and 8 dimensions respectively,

$$
\begin{aligned}
& \Omega_{\mathrm{HCS}}^{(3,6)}=-2 \eta^{2} \Omega_{\mathrm{CS}}^{(3)}-\varepsilon^{\mu \nu \lambda} \operatorname{Tr} \gamma_{5} D_{\lambda} \Phi\left(F_{\mu \nu} \Phi+F_{\mu \nu} \Phi\right) . \\
& \Omega_{\mathrm{HCS}}^{(3,8)}=6 \eta^{4} \Omega_{\mathrm{CS}}^{(3)}-\varepsilon^{\mu \nu \lambda} \operatorname{Tr} \gamma_{5}\left\{6 \eta^{2}\left(\Phi D_{\lambda} \Phi-D_{\lambda} \Phi \Phi\right) F_{\mu \nu}\right. \\
&\left.\quad-\left[\left(\Phi^{2} D_{\lambda} \Phi \Phi-\Phi D_{\lambda} \Phi \Phi^{2}\right)-2\left(\Phi^{3} D_{\lambda} \Phi-D_{\lambda} \Phi \Phi^{3}\right)\right] F_{\mu \nu}\right\} .
\end{aligned}
$$

Note that the leading term in both (9) and (10) is the CS density (3).

The HCS density in $d=5$ arrived at from the one-step descent of the HCP density in $D=6$, itself descended from the CP density in 8 dimensions, is

$$
\Omega_{\mathrm{HCS}}^{(5,8)}=2 \eta^{2} \Omega_{\mathrm{CS}}^{(5)}+\varepsilon^{\mu \nu \rho \sigma \lambda} \operatorname{Tr} \gamma_{7}\left[D_{\lambda} \Phi\left(\Phi F_{\mu \nu} F_{\rho \sigma}+F_{\mu \nu} \Phi F_{\rho \sigma}+F_{\mu \nu} F_{\rho \sigma} \Phi\right)\right]
$$

and the HCS density in $d=7$ arrived at from the one-step descent of the HCP density in $D=8$, itself descended from the CP density in 10 dimensions, is

$$
\Omega_{\mathrm{HCS}}^{(7,10)}=\eta^{2} \Omega_{\mathrm{CS}}^{(7)}+\varepsilon^{\mu \nu \rho \sigma \tau \lambda \kappa} \operatorname{Tr} \gamma_{9} D_{\kappa} \Phi F_{\mu \nu} F_{\rho \sigma}\left(F_{\tau \lambda} \Phi+\Phi F_{\tau \lambda}\right) .
$$

Note that the leading term in (11) is the CS density (4), and that in (12) the CS density (5).

Thus, in all odd dimensions where both a CS and a HCS density exist, the leading term in the HCS density $\Omega_{\mathrm{HCS}}^{(d, N)}$ in (odd) $d$ dimensions that pertains to the HCP density desecnded from the CP density in (even) $N$ dimensions, is the CS density $\Omega_{\mathrm{CS}}^{(d)}$.

The situation is as expected, entirely different for HCS densities in even dimensions, where there are no usual CS densities. In those cases the HCS densities are expressed entirely in terms of the Higgs scalar $\Phi$, its covariant derivative $D_{\mu} \Phi$, and of course the curvature $F_{\mu \nu}$.

Here, we display only the HCS densities in $d=4$, arrived at from the one-step descents of the HCP densities in $D=5$, each of them descended from the CP densities in 6 and 8 dimensions respectively,

$$
\begin{aligned}
\Omega_{\mathrm{HCS}}^{(4,6)}= & \varepsilon^{\mu \nu \rho \sigma} \operatorname{Tr} F_{\mu \nu} F_{\rho \sigma} \Phi \\
\Omega_{\mathrm{HCS}}^{(4,8)}= & \varepsilon^{\mu \nu \rho \sigma} \operatorname{Tr}\left[\Phi\left(\eta^{2} F_{\mu \nu} F_{\rho \sigma}+\frac{2}{9} \Phi^{2} F_{\mu \nu} F_{\rho \sigma}+\frac{1}{9} F_{\mu \nu} \Phi^{2} F_{\rho \sigma}\right)\right. \\
& \left.\quad-\frac{2}{9}\left(\Phi D_{\mu} \Phi D_{\nu} \Phi-D_{\mu} \Phi \Phi D_{\nu} \Phi+D_{\mu} \Phi D_{\nu} \Phi \Phi\right) F_{\rho \sigma}\right] .
\end{aligned}
$$


Finally, we select the gauge group appropriate for the purpose of transiting from Yang-Mills to gravity. As stated at the outset by (11), this group is $S O(D)(D=2 n)$, the orthogonal group of the non-Abelian field defining the Chern-Pontryagin (CP) density from which the Chern-Simons (CS) density is derived, and in the case of Higgs-CS, the gauge group of the Higgs-CP density from which the HCS is derived.

In the case of CS densities the Dirac matrix representations, e.g. (2), are employed, such that the spin-connection and the $S O(D)$ YM connections are identified, $A_{\mu}^{a b}=\omega_{\mu}^{a b}$.

The situation is somewhat more involved in the case of Higgs-CS (HCS) densities, in which case we have HCS densities both in odd deimensions, e.g. (9), (10), (11), (12) and (3), and in even dimensions $e, g$. (13) and (14). Here, $D$ is even for the HCS in odd dimensions but it is even when the HCS is in even dimensions. Besides, in this case the multiplicity of the Higgs scalar $\Phi$ must alo be chosen 5 . For HCS densities in odd dimensions, i.e. with even D, (11) is augmented by the choice of Higgs multiplet,

$$
A_{\mu}=-\frac{1}{2} A_{\mu}^{a b} \gamma_{a b}, \quad \text { and } \quad \Phi=2 \phi^{a} \gamma_{a, D+1}
$$

while for HCS densities in even dimensions, i.e. with $D$ odd,

$$
A_{\mu}=-\frac{1}{2} A_{\mu}^{a b} \Sigma_{a b}, \quad \text { and } \quad \Phi=2 \phi^{a} \Sigma_{a, D+1}
$$

where $\Sigma_{a b}^{( \pm)}$are one or other chiral representations of $S O(D)$

$$
\Sigma_{a b}^{( \pm)}=\frac{1}{2}\left(\mathbb{I} \pm \gamma_{D+1}\right) \gamma_{a b}, \quad a=1,2, \ldots D
$$

It is in order to remark that for odd $d$ only the CS densities $\Omega_{\mathrm{CS}}^{(d)}$, (3)-(5)), appear in the HCS

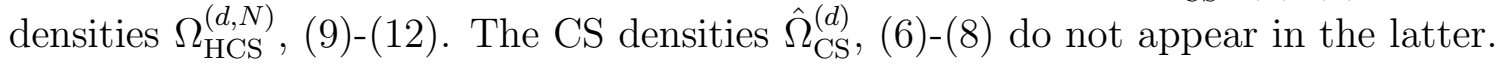

\section{Chern-Simons gravity (CSG) and Higgs-CS gravity (HCSG)}

Both the CSG and the HCSG result in gravitational systems consisting of the superposition of $p$-Einstein-Hilbert densities (22) which we present here to be self-comtained and to fix the notation.

In terms of the the spin-connection $\omega_{M}^{a b}$, the covariant derivative of (some frame-vector valued field) $\phi^{a}$ is defined as

$$
D_{M} \phi^{a}=\partial_{M} \phi^{a}+\omega_{M}^{a b} \phi^{b}
$$

and employing further the vielbein field $e_{M}^{a}$ there follow the definitions of the gravitational curvature and torsion

$$
\begin{aligned}
& R_{\mu \nu}^{a b}=\left(D_{[\mu} D_{\nu]}\right)^{a b}=\partial_{\mu} \omega_{\nu}^{a b}-\partial_{\nu} \omega_{\mu}^{a b}+\omega_{\mu}^{a c} \omega_{\nu}^{c b}-\omega_{\nu}^{a c} \omega_{\mu}^{c b} \\
& C_{\mu \nu}^{a}=D_{[\mu} e_{\nu]}^{a}=\partial_{\mu} e_{\nu}^{a}-\partial_{\nu} e_{\mu}^{a}+\omega_{\mu}^{a c} e_{\nu}^{c}-\omega_{\nu}^{a c} e_{\mu}^{c},
\end{aligned}
$$

\footnotetext{
${ }^{5}$ These choices coincide with those for which monopoles on $\mathbb{R}^{D}$ are constructed [12.
} 
with

$$
\mu=1,2, \ldots, d ; \quad a=1,2, \ldots, d .
$$

To define the $p$-Einsten-Hilbert 6 ( $p$-EH) Lagrangians, we split the indices on the Levi-Civita symbols as follows

$$
\varepsilon^{\mu_{1} \mu_{2} \ldots \mu_{2 p} \mu_{2 p+1} \ldots \mu_{d}} \quad \text { and } \quad \varepsilon_{a_{1} a_{2} \ldots a_{2 p} a_{2 p+1} \ldots a_{d}}
$$

such that in $d$-dimensional spacetime the Lagrangians are

$$
\mathcal{L}_{\mathrm{EH}}^{(p, d)}=\varepsilon^{\mu_{1} \mu_{2} \ldots \mu_{2 p} \mu_{2 p+1} \ldots \mu_{d}} e_{\mu_{2 p+1}}^{a_{2 p+1}} e_{\mu_{2 p+2}}^{a_{2 p+2}} \ldots e_{\mu_{d}}^{a_{d}} \varepsilon_{a_{1} a_{2} \ldots a_{2 p} a_{2 p+1} \ldots a_{d}} R_{\mu_{1} \mu_{2}}^{a_{1} a_{2}} R_{\mu_{3} \mu_{4}}^{a_{3} a_{4}} \ldots R_{\mu_{2 p-1} \mu_{2 p}}^{a_{2 p-1} a_{2 p}}
$$

For $p=0$ in $d=2 p$ this is a total divergence, while for $d \geq 2 p$ it is the cosmological constant. For $p=1$ it is the usual Einstein-Hilbert (EH) Lagrangian in $d$-dimensions, for $p=2$ it is the usual Gauss-Bonnet Lagrangian in $d$-dimensions, etc.

The definitions of (22) include the Levi-Civita symbol in both the frame indices and the coordinate indices. Thus it is appropriate to adopt the definitions (3)-(5) for the CS densities since in that case evaluating the traces will result in some superpositions of (usual) $p$-EH Lagrangians. In this respect, the choice of (9)-(12) for the HCS densities is the appropriate one. Examples of CSG and HCSG systems are given in the next Subsections, respectively.

\subsection{Chern-Simons gravity (CSG): odd dimensions}

As remarked earlier, the prescription (1) for transiting from YM to gravity in $d$ dimensions, yield a frame-index $a=1,2, \ldots d+1$ which is defective. This defect is overcome by splitting $a$ as $a=(\alpha, d+1)=(\alpha, D)$, with $\alpha=1,2, \ldots, d$, as will be described in the following Subsections.

In this case the prescription (11), or the first member of (15)), is refined as follows,

$$
A_{\mu}=-\frac{1}{2} \omega_{\mu}^{\alpha \beta} \gamma_{\alpha \beta}+\kappa e_{\mu}^{\alpha} \gamma_{\alpha D} \Rightarrow F_{\mu \nu}=-\frac{1}{2}\left(R_{\mu \nu}^{\alpha \beta}-\kappa^{2} e_{[\mu}^{\alpha} e_{\nu]}^{\beta}\right) \gamma_{\alpha \beta}+\kappa C_{\mu \nu}^{\alpha} \gamma_{\alpha D}
$$

where $C_{\mu \nu}^{\alpha}=D_{[\mu} e_{\nu]}$ is the torsion. Clearly, $\alpha$ is now the frame-index with the correct range. The constant $\kappa$ in (23) has dimensions $L^{-1}$ to compensate for the difference in the dimensions of the connection and the Vielbein.

Substituting (23) in $\Omega_{\mathrm{CS}}^{n}$, (33)-(5), yields the CSG models in $d=3,5,7$,

$$
\begin{aligned}
\mathcal{L}_{\mathrm{CSG}}^{(3)}= & -\kappa \varepsilon^{\mu \nu \lambda} \varepsilon_{a b c}\left(R_{\mu \nu}^{a b}-\frac{2}{3} \kappa^{2} e_{\mu}^{a} e_{\nu}^{b}\right) e_{\lambda}^{c} \\
\mathcal{L}_{\mathrm{CSG}}^{(5)}= & \kappa \varepsilon^{\mu \nu \rho \sigma \lambda} \varepsilon_{a b c d e}\left(\frac{3}{4} R_{\mu \nu}^{a b} R_{\rho \sigma}^{c d}-\kappa^{2} R_{\mu \nu}^{a b} e_{\rho}^{c} e_{\sigma}^{d}+\frac{3}{5} \kappa^{4} e_{\mu}^{a} e_{\nu}^{b} e_{\rho}^{c} e_{\sigma}^{d}\right) e_{\lambda}^{e} \\
\mathcal{L}_{\mathrm{CSG}}^{(7)}= & -\kappa \varepsilon^{\mu \nu \rho \sigma \tau \kappa \lambda} \varepsilon_{a b c d e f g}\left(\frac{1}{8} R_{\mu \nu}^{a b} R_{\rho \sigma}^{c d} R_{\tau \kappa}^{e f}-\frac{1}{4} \kappa^{2} R_{\mu \nu}^{a b} R_{\rho \sigma}^{c d} e_{\tau}^{e} e_{\kappa}^{f}\right. \\
& \left.+\frac{3}{10} \kappa^{4} R_{\mu \nu}^{a b} e_{\rho}^{c} e_{\sigma}^{d} e_{\tau}^{e} e_{\kappa}^{f}-\frac{1}{7} \kappa^{6} e_{\mu}^{a} e_{\nu}^{b} e_{\rho}^{c} e_{\sigma}^{d} e_{\tau}^{e} e_{\kappa}^{f}\right) e_{\lambda}^{g}
\end{aligned}
$$

\footnotetext{
${ }^{6}$ Aka. Lovelock gravitity.
} 
Each of these is a linear sum of all the $p$-Einstein-Hilbert $(\mathrm{EH})$ Lagrangians $\mathcal{L}_{\mathrm{EH}}^{(p, d)}$ defined in the given dimension $d$ (the $p=0$ member being the cosmological constant term.).

In the notation of Appendix A,

$$
\begin{aligned}
\mathcal{L}_{\mathrm{CSG}}^{(3)} & =-\kappa\left[\tau_{(1)} \mathcal{L}_{\mathrm{EH}}^{(1,3)}-\tau_{(0)} \kappa^{2} \mathcal{L}_{\mathrm{EH}}^{(0,3)}\right] \\
\mathcal{L}_{\mathrm{CSG}}^{(5)} & =\kappa\left[\tau_{(2)} \mathcal{L}_{\mathrm{EH}}^{(2,5)}-\tau_{(1)} \kappa^{2} \mathcal{L}_{\mathrm{EH}}^{(1,5)}+\tau_{(0)} \kappa^{4} \mathcal{L}_{\mathrm{EH}}^{(0,5)}\right] \\
\mathcal{L}_{\mathrm{CSG}}^{(7)} & =-\kappa\left[\tau_{(3)} \mathcal{L}_{\mathrm{EH}}^{(3,7)}-\tau_{(2)} \kappa^{2} \mathcal{L}_{\mathrm{EH}}^{(2,7)}+\tau_{(1)} \kappa^{4} \mathcal{L}_{\mathrm{EH}}^{(1,7)}-\tau_{(0)} \kappa^{6} \mathcal{L}_{\mathrm{EH}}^{(0,7)}\right]
\end{aligned}
$$

where the dimensionless constants $\tau_{(p)}$ can be read off (24), (25) and (26) .

\subsection{Higgs-Chern-Simons gravity (HCSG): all dimensions}

The HCS densities, displayed in Subsection 2.2, involve both the YM and the Higgs fields. The passage of the YM-Higgs (YMH) system to gravity is prescribed by (15) in odd dimensions, and (16) in even. We will henceforth refer to the case of odd $d=D-1$, namely to the prescription (23), since for even $d$ the appropriate prescription can be read off (23) by formally replacing $\left(\gamma_{\alpha \beta}, \gamma_{\alpha D}\right)$ with $\left(\Sigma_{\alpha \beta}, \Sigma_{\alpha D}\right)$, the latter defined by (17).

As in the previous Subsection, the index $a=(\alpha, D)$ is split such that $\alpha$ now is the frame-index, and the refined version of the second mamber of (15) we apply is

$$
\begin{aligned}
2^{-1} \Phi & =\left(\phi^{\alpha} \gamma_{\alpha, D+1}+\phi \gamma_{D, D+1}\right) \Rightarrow \\
& \Rightarrow 2^{-1} D_{\mu} \Phi=\left(D_{\mu} \phi^{\alpha}-\kappa e_{\mu}^{\alpha} \phi\right) \gamma_{\alpha, D+1}+\left(\partial_{\mu} \phi+\kappa e_{\mu}^{\alpha} \phi^{\alpha}\right) \gamma_{D, D+1}
\end{aligned}
$$

where

$$
D_{\mu} \phi^{\alpha}=\partial_{\mu} \phi^{\alpha}+\omega_{\mu}^{\alpha \beta} \phi^{\beta}
$$

is the gravitational covariant derivative.

We employ (23) and (30) to calculate the traces in the HCS formulas in Subsection 2.2. Here, we display only the pair of HCSG (gravitational) systems arising from HCS densities (9)-(10) in $d=3$, and the pair arising from (13)-(14) in $d=4$.

The pair in $d=3$ is

$$
\begin{aligned}
& \mathcal{L}_{\mathrm{HCSG}}^{(3,6)}= \varepsilon^{\lambda \mu \nu} \varepsilon_{\alpha \beta \gamma}\left\{2 \eta^{2} \kappa\left(e_{\lambda}^{\gamma} R_{\mu \nu}^{\alpha \beta}-\frac{2}{3} \kappa^{2} e_{\mu}^{\alpha} e_{\nu}^{\beta} e_{\lambda}^{\gamma}\right)\right. \\
&-\left[2\left(R_{\mu \nu}^{\alpha \beta}-\kappa^{2} e_{[\mu}^{\alpha} e_{\nu]}^{\beta}\right)\left[\phi^{\gamma}\left(\partial_{\lambda} \phi+\kappa e_{\lambda}^{d} \phi^{\delta}\right)-\phi\left(D_{\lambda} \phi^{\gamma}-\kappa e_{\lambda}^{\gamma} \phi\right)\right]\right. \\
&\left.\left.-4 \kappa \phi^{\alpha}\left(D_{\lambda} \phi^{\beta}-\kappa e_{\lambda}^{\beta} \phi\right) C_{\mu \nu}^{\gamma}\right]\right\} \\
& \mathcal{L}_{\mathrm{HCSG}}^{(3,8)}=-3 \eta^{2} \mathcal{L}_{\mathrm{HGCS}}^{(3,3)}-12 \varepsilon^{\lambda \mu \nu} \varepsilon_{\gamma \alpha \beta}\left[\eta^{2}-\left(\left|\phi^{\delta}\right|^{2}+\phi^{2}\right)\right] \cdot \\
& \cdot\left[\left[\phi^{\gamma}\left(\partial_{\lambda} \phi+\kappa e_{\lambda}^{d} \phi^{\delta}\right)-\phi\left(D_{\lambda} \phi^{\gamma}-\kappa e_{\lambda}^{\gamma} \phi\right)\right]\right. \\
&\left.-4 \kappa \phi^{\alpha}\left(D_{\lambda} \phi^{\beta}-\kappa e_{\lambda}^{\beta} \phi\right) C_{\mu \nu}^{\gamma}\right]
\end{aligned}
$$


and the pair in $d=4$ is

$$
\begin{aligned}
\mathcal{L}_{\mathrm{HCSG}}^{(4,6)}= & -\varepsilon^{\mu \nu \rho \sigma} \varepsilon_{\alpha \beta \gamma \delta} \phi\left[R_{\mu \nu}^{\alpha \beta} R_{\rho \sigma}^{\gamma \delta}-4 \kappa^{2} e_{\rho}^{\gamma} e_{\sigma}^{\delta} R_{\mu \nu}^{\alpha \beta}+4 \kappa^{4} e_{\mu}^{\alpha} e_{\nu}^{\beta} e_{\rho}^{\gamma} e_{\sigma}^{\delta}\right] \\
& +2 \kappa \varepsilon^{\mu \nu \rho \sigma} \varepsilon_{\alpha \beta \gamma \delta}\left(R_{\mu \nu}^{\alpha \beta}-\kappa^{2} e_{[\mu}^{\alpha} e_{\nu]}^{\beta}\right) C_{\rho \sigma}^{\gamma} \phi^{\delta} \\
\mathcal{L}_{\mathrm{HCSG}}^{(4,8)}= & {\left[\eta^{2}-\frac{1}{3}\left(\left|\phi^{\alpha}\right|^{2}+\phi^{2}\right)\right] \mathcal{L}_{\mathrm{HGCS}}^{(4,6)}-} \\
& -\frac{2}{3} \varepsilon^{\mu \nu \rho \sigma} \varepsilon_{\alpha \beta \gamma \delta}\left[\left(R_{\mu \nu}^{\alpha \beta}-\kappa^{2} e_{[\mu}^{\alpha} e_{\nu]}^{\beta}\right) \phi_{\rho}^{\gamma}\left(\phi \phi_{\sigma}^{\delta}-2 \phi^{\delta} \phi_{\sigma}\right)+\frac{2}{3} C_{\mu \nu}^{\alpha} \phi^{\beta} \phi_{\rho}^{\gamma} \phi_{\sigma}^{\delta}\right]
\end{aligned}
$$

where we have used an abbreviated notation

$$
\phi_{\mu}=\partial_{\mu} \phi, \quad \phi_{\mu}^{\alpha}=D_{\mu} \phi^{\alpha}, \quad \mu=1,2, \ldots d, \quad \alpha=1,2, \ldots d .
$$

Some concluding remarks are now in order. We observe the following qualitative properties of the listed HCSG (gravitaional) systems:

- In the pair of models in $d=3$, namely (32)-(33), the leading terms are the usual EinsteinHilbert Lagrangian $\mathcal{L}_{\mathrm{EH}}^{(1,3)}$, viz. (24) or (27). By contrast, in (니) and (35), no purely gravitational Lagrangians $\mathcal{L}_{\mathrm{EH}}^{(p, d)}$ appear without the presence of the frame-vector field $\phi^{\alpha}$ and the scalar $\phi$. This is not surprising, since there exist no CS densities in even dimensions.

- The models $\mathcal{L}_{\mathrm{HGCS}}^{(d, N)}$ pertaining to higher values of $N$ in the HCS densities $\Omega_{\mathrm{HCS}}^{(d, N)}$ from which they follow, feature Lagrangians with lower $N$, nested inside.

- All HCSG models, in odd and even dimensions, feature the torsion term explicitly. This, together with the fact that they feature the gravitational covariant derivative (31), means that these models can sustain non-zero torsion. Whether or not torsion-free solutions may exist, must be checked in each case.

- The frame-vector field $\phi^{\alpha}$ and the scalar $\phi$ are relics of the Higgs scalar in the YangMills-Higgs systems giving rise to the HCSG models. Thus we would expect that these are gravitational coordinates and not matter fields that might result in hairy solutions. Accordingly, we would expect that these models support only black hole solutions, and not regular ones.

\section{Gravitational CS (GCS) densities}

Gravitational CS (GCS) densities, as their name suggests, are not gravitational models like the CSG and HCSG models discussed above. They are the analogues of the non-Abelian (nA) CS densities, that are employed in various applications of nA gauge theories. Like the latter, the GCS densities are designed to find application in the same way, in gravitational theories.

Together with the CSG models discussed above, GCS densities are derived from the nA CS densities by applying the prescription (1), but not in the refined versions (23). As result the 
frame indices $a=1,2, \ldots, D, D=d+1$, in the resulting gravitational density have the wrong range, namely that they range over $\alpha=1,2, \ldots, d$. This defect is corrected by introducing a (rather aribitary) truncation, which consists of setting some components of the spin-connection $\omega_{\mu}^{a b}=\left(\omega_{\mu}^{\alpha \beta}, \omega_{\mu}^{\alpha, D}\right)$ equal to zero by hand according to

$$
\omega_{\mu}^{\alpha, D}=0 \quad \Rightarrow \quad R_{\mu \nu}^{\alpha, D}=0 .
$$

The resulting density is expressed exclusively in terms of the components of the (gravitational) connection and curvature $\left(\omega_{\mu}^{\alpha \beta}, R_{\mu \nu}^{\alpha \beta}\right)$, such that now the frame-indices $\alpha$ transform with the required group $S O(d)$ and not $S O(D)$. This is adopted as the definition of gravitational ChernSimons (GCS) density.

As in Section 3, one has again the choice 7 of opting for the definitions (3)-(15), or, (6) -(8) for the nA CS densities, which prior to implementing the truncation (36) are the CS densities for gauge group $S O(D)$. A further important distinction form the nA case arises here in the gravitational case when the choice (6) -(8) is made for the nA CS densities. As a result of gammamatrix identities 8, it turns ot that substituting (10)-(2) in (6)-(8), these traces vanish itentically in all $4 p-3$ dimensions. As a result, with this choice gravitational CS (GCS) densities can be constructed only in $4 p-1$, and not, all odd dimensions. The choice of ([6)-(8) is for the nA CS densities on the other hand, is not subject to this obstacle and it affords the definition of CSG densities in all odd dimensions. In this case the resulting CSG density will feature the Levi-Civita symbol with frame indices, which subject to the truncation (36), collapses.

Succinctly stated, CSG densities thus constructed, exist in $4 p-1$ dimensionst only. Applying the prescription in $d=3,5,7$,

$$
A_{\mu}=-\frac{1}{2} \omega_{\mu}^{a b} \gamma_{a b}, \quad a=1,2, \ldots, d+1,
$$

namely by eveluating the traces in (66)-(8) and then implementing the truncation (36),

$$
\begin{aligned}
\hat{\Omega}_{\mathrm{GCS}}^{(3)}= & -\frac{1}{2 \cdot 2 !} \varepsilon^{\lambda \mu \nu} \delta_{\alpha \beta}^{\bar{\alpha} \bar{\beta}} \omega_{\lambda}^{\alpha \beta}\left[R_{\mu \nu}^{\bar{\alpha} \bar{\beta}}-\frac{2}{3}\left(\omega_{\mu} \omega_{\nu}\right)^{\bar{\alpha} \bar{\beta}}\right] \\
\hat{\Omega}_{\mathrm{GCS}}^{(5)}= & 0 \\
\hat{\Omega}_{\mathrm{GCS}}^{(7)}= & \frac{1}{2 \cdot 6 !} \varepsilon^{\lambda \mu \nu \rho \sigma \tau \kappa} \hat{\delta}_{\alpha \beta \gamma \delta}^{\bar{\alpha} \bar{\beta} \bar{\gamma} \bar{\delta}} \omega_{\lambda}^{\alpha \beta}\left[R_{\mu \nu}^{\gamma \delta} R_{\rho \sigma}^{\bar{\alpha} \bar{\beta}} R_{\tau \kappa}^{\bar{\gamma} \bar{\delta}}-\frac{4}{5} R_{\mu \nu}^{\gamma \delta} R_{\rho \sigma}^{\bar{\alpha} \bar{\beta}}\left(\omega_{\tau} \omega_{\kappa}\right)^{\bar{\gamma} \bar{\delta}}-\frac{2}{5} R_{\mu \nu}^{\gamma \delta}\left(\omega_{\rho} \omega_{\sigma}\right)^{\bar{\alpha} \bar{\beta}} R_{\tau \kappa}^{\bar{\gamma} \bar{\delta}}\right. \\
& \left.\quad+\frac{4}{5} R_{\mu \nu}^{\gamma \delta}\left(\omega_{\rho} \omega_{\sigma}\right)^{\bar{\alpha} \bar{\beta}}\left(\omega_{\tau} \omega_{\kappa}\right)^{\bar{\gamma} \bar{\delta}}-\frac{8}{35}\left(\omega_{\mu} \omega_{\nu}\right)^{\gamma \delta}\left(\omega_{\rho} \omega_{\sigma}\right)^{\bar{\alpha} \bar{\beta}}\left(\omega_{\tau} \omega_{\kappa}\right)^{\bar{\gamma} \bar{\delta}}\right]
\end{aligned}
$$

\footnotetext{
${ }^{7}$ Recall that previously in the derivation of the CSG models, the choice of (3)-(5) was made since the LeviCivita symbol with frame indices, which results from the presence of the chiral matrix $\gamma_{D, D+1}$ under the trace, was required for the description of gravitational systems. Here, we have no such constraint.

${ }^{8}$ The identity in question, in $2 n$ dimensions is$$
\gamma_{a_{1} a_{2} \ldots a_{n} b_{1} b_{2} \ldots b_{2 n}}=\delta_{a_{1} a_{2} \ldots a_{n}}^{b_{1} b_{2} \ldots b_{2 n}} \mathbb{I}+\varepsilon_{a_{1} a_{2} \ldots a_{n} b_{1} b_{2} \ldots b_{2 n}} \gamma_{2 n+1}
$$

where $\gamma_{a_{1} a_{2} \ldots a_{n} b_{1} b_{2} \ldots b_{2 n}}$ is the totally antisymmetrised product of $2 n$ gamma matrices in $2 n$ dimensions, and $\gamma_{2 n+1}$ is the chiral matrix.
} 
etc., where the symbol $\hat{\delta}_{\alpha \beta \gamma \delta}^{\bar{\alpha} \bar{\beta} \bar{\gamma}}$ in (40) is

$$
\hat{\delta}_{\alpha \beta \gamma \delta}^{\bar{\alpha} \bar{\beta} \bar{\gamma} \bar{\delta}}=\frac{1}{9} \delta_{\alpha \beta \gamma \delta}^{\bar{\alpha} \bar{\beta} \bar{\gamma} \bar{\delta}}+\frac{1}{4} \delta_{\alpha \beta}^{\gamma \delta} \delta_{\bar{\alpha} \bar{\beta}}^{\bar{\gamma} \bar{\delta}}
$$

\subsection{Gravitational Higgs-CS (GHCS) densities}

The main purpose of constructing GHCS densities would be to supply GCS densities in both odd and even dimensions, possibly including in $4 p-3$ dimensions which were absent in the Higgs free case above. Thus here too we employ the Higgs-CS (HCS) densities presented in Section 2.2.

In Section 3, where CS and HCS gravities were constructed, it turned out that in all odd dimensions the leading term in the HCS gravity (HCSG) was the CS gravity (CSG). In that case the choice of CS densities (3)-(15) displaying the chiral matrix $\gamma_{D+1}=\left(\gamma_{d+2}\right)$, was made with the aim of generating a gravitational model, which coincided with (9)-(12), the defining of the HCS densities in odd dimensions.

If we invoke the same criterion here as in the construction of HCS gravities (HCSG), namely that the leading terms in the GHCS densities in $4 p-1$ dimensions be the CSG densities, e.g. $\hat{\Omega}_{\mathrm{CS}}^{(d)}$ given by (6) and (8) in $d=3,7$, then this is achieved by deforming (9)-(10) and (12), by removing the chiral matrix under the trace, by hand.

The corresponding consideration in $d=4 p-3$ dimensions fails to yield a nontrivial result. We know the GCS density $\hat{\Omega}_{\mathrm{CS}}^{(5)}$ vanishes, $c f$. (7).

To illustrate these points, consider the examples of proposed (deformed) HCS densities in $d=3,5$

$$
\begin{aligned}
& \hat{\Omega}_{\mathrm{HCS}}^{(3,6)}=-2 \eta^{2} \hat{\Omega}_{\mathrm{CS}}^{(3)}-\varepsilon^{\mu \nu \lambda} \operatorname{Tr} D_{\lambda} \Phi\left(F_{\mu \nu} \Phi+F_{\mu \nu} \Phi\right) \\
& \hat{\Omega}_{\mathrm{HCS}}^{(5,8)}=2 \eta^{2} \hat{\Omega}_{\mathrm{CS}}^{(5)}+\varepsilon^{\mu \nu \rho \sigma \lambda} \operatorname{Tr} D_{\lambda} \Phi\left(\Phi F_{\mu \nu} F_{\rho \sigma}+F_{\mu \nu} \Phi F_{\rho \sigma}+F_{\mu \nu} F_{\rho \sigma} \Phi\right)
\end{aligned}
$$

Applying the prescription

$$
A_{\mu}=-\frac{1}{2} \omega_{\mu}^{a b} \gamma_{a b}, \quad \text { and } \quad \Phi=2 \phi^{a} \gamma_{a, d+2}, \quad a=1,2, \ldots, d+1
$$

and then implementing the truncation(36), we find the two GHCS densities

$$
\begin{aligned}
& \hat{\Omega}_{\mathrm{GHCS}}^{(3,6)}=-2 \eta^{2} \hat{\Omega}_{\mathrm{GCS}}^{(3)}+4 \varepsilon^{\lambda \mu \nu} \phi^{\alpha} R_{\mu \nu}^{\alpha \beta} D_{\lambda} \phi^{\beta} \\
& \hat{\Omega}_{\mathrm{GHCS}}^{(5,8)}=0+0
\end{aligned}
$$

As we see from (46), the gravitational HCS (GHCS) densities vanish in $4 p-3$ dimensions, just as the gravitational CS (GCS) densities, for the same technical reason. (Not deforming the HCS density by removing the chiral matrix from under the trace does not change the situation. In that case the Levi-Civita symbol in the frame indices in $D=d+1$ dimensions appear, which vanish when the trauncation (36) is implemented.)

Concerning the construction of GHCS densities in even dimensions, we employ the HCS densities $\Omega_{\text {GHCS }}^{(4,6)}$ and $\Omega_{\text {GHCS }}^{(4,8)}$ given by $(\underline{13})$-(14) in $d=4$. The prescription applied here is also 
(44), but with $\gamma_{a b}$ formally replaced by $\Sigma_{a b}, c f$. (17), followed by the truncation (36). The result is

$$
\begin{aligned}
& \hat{\Omega}_{\mathrm{GHCS}}^{(4,6)}=-\frac{1}{4} \varepsilon^{\mu \nu \rho \sigma} R_{\mu \nu}^{\alpha \beta} R_{\rho \sigma}^{\alpha \beta} \phi \\
& \hat{\Omega}_{\mathrm{GHCS}}^{(4,8)}=-\varepsilon^{\mu \nu \rho \sigma} R_{\mu \nu}^{\alpha \beta}\left\{\left[\frac{1}{8}\left(1-\frac{1}{3}\left|\phi^{a}\right|^{2}\right) R_{\rho \sigma}^{\alpha \beta}+\frac{1}{3} \phi_{\rho \sigma}^{\alpha \beta}\right] \phi+\frac{4}{3} \phi^{\alpha} D_{\rho} \phi^{\beta} \partial_{\sigma} \phi\right\}
\end{aligned}
$$

where the abbreviated notation

$$
\phi_{\mu \nu}^{\alpha \beta}=D_{[\mu} \phi^{\alpha} D_{\nu]} \phi^{\beta}, \quad \text { and } \quad \phi=\phi^{5} .
$$

In even dimensions, there are no exclusions like in odd dimensions, and GHCS densities like (47) and (48) exist in all even dimensions.

Some concluding remarks are in order here.

- In odd dimensions, gravitational CS (GCS) densities and gravitational HCS (GHCS) densities are defined only in $4 p-1$ and not in $4 p-3$ dimensions.

- In $4 p-1$ dimensions, the leading term in the GHCS density is the GCS density.

- Gravitational HCS (GHCS) densities can be defined in all even dimensions where no GCS densities exist.

- As in the case of HCS gravities (HCSG), the frame-vector field $\phi^{\alpha}$ and the scalar $\phi$ are gravitational degrees of freedom.

- If employed as CS densities to modify a gravitational model, the GHCS densities would be applied to HCSG gravitational models decsribed in Section 3, which are decsribed by the same gravitational fields. This is because the fields $\phi^{a}=\left(\phi^{\alpha}, \phi\right)$ are gravitational degrees of freedom and their dynamics is given naturally by the HCSG models in the given dimension.

\section{Summary}

An illustraive presentation of Chern-Simons gravities in all dimensions, and gravitational ChernSimons densities in all even and in $4 p-1$ odd dimensions is given. These "Chern-Simons densities" are one-step descendents of (Higgs-)Chern-Pontryagin densities defined in all dimensions, and which result from the dimensional decsent of a Chern-Pontryagin density in some (higher) even dimension. A distiction is made between CS gravitational systems and the CS densities, and each is presented separately followed by comments in its own Section.

Acknowledgements: My deepest gratitude to Eugen Radu for his unstinting support in preparing this report. My thanks to Friedrich Hehl for having introduced me to the Einstein-Cartan formulation, and to Ruben Manvelyan for helpful extended discussions. Thanks to Jorge Zanelli

for helpful correspondence. 


\section{References}

[1] E. Witten, Nucl. Phys. B 311 (1988) 46. doi:10.1016/0550-3213(88)90143-5

[2] A. H. Chamseddine, Phys. Lett. B 233 (1989) 291. doi:10.1016/0370-2693(89)91312-9

[3] A. H. Chamseddine, Nucl. Phys. B 346 (1990) 213. doi:10.1016/0550-3213(90)90245-9

[4] S. Deser, R. Jackiw and S. Templeton, Phys. Rev. Lett. $48 \quad$ (1982) 975. doi:10.1103/PhysRevLett.48.975

[5] R. Araneda, R. Aros, O. Miskovic and R. Olea, Phys. Rev. D 93 (2016) no.8, 084022 doi:10.1103/PhysRevD.93.084022 [arXiv:1602.07975 [hep-th]].

[6] S. Deser, R. Jackiw and S. Templeton, Annals Phys. 140 (1982) 372 [Annals Phys. 281 (2000) 409] Erratum: [Annals Phys. 185 (1988) 406]. doi:10.1006/aphy.2000.6013, 10.1016/0003-4916(82)901646

[7] A. Mardones and J. Zanelli, Class. Quant. Grav. 8 (1991) 1545. doi:10.1088/0264-9381/8/8/018

[8] J. Zanelli, Class. Quant. Grav. 29 (2012) 133001 doi:10.1088/0264-9381/29/13/133001 arXiv:1208.3353 [hep-th]].

[9] S. W. MacDowell and F. Mansouri, Phys. Rev. Lett. 38 (1977) 739 Erratum: [Phys. Rev. Lett. 38 (1977) 1376]. doi:10.1103/PhysRevLett.38.1376, 10.1103/PhysRevLett.38.739

[10] R. Jackiw and S. Y. Pi, Phys. Rev. D 68 (2003) 104012 doi:10.1103/PhysRevD.68.104012 gr-qc/0308071.

[11] see for example, R. Jackiw, "Chern-Simons terms and cocycles in physics and mathematics", in E.S. Fradkin Festschrift, Adam Hilger, Bristol (1985).

[12] T. Tchrakian, J. Phys. A 44 (2011) 343001 doi:10.1088/1751-8113/44/34/343001 arXiv:1009.3790 [hep-th]].

[13] E. Radu and T. Tchrakian, doi:10.1142/9789814440349-0020 arXiv:1101.5068 [hep-th].

[14] D. H. Tchrakian, J. Phys. A 48 (2015) no.37, 375401 doi:10.1088/1751-8113/48/37/375401 arXiv:1505.05344 [hep-th]].

[15] P. Forgacs and N. S. Manton, Commun. Math. Phys. 72 (1980) 15. doi:10.1007/BF01200108

[16] A. S. Schwarz and Y. S. Tyupkin, Nucl. Phys. B 187 (1981) 321. doi:10.1016/0550-3213(81)90277-7

[17] D. Kapetanakis and G. Zoupanos, Phys. Rept. 219 (1992) 4. doi:10.1016/0370-1573(92)90101-5

[18] T. N. Sherry and D. H. Tchrakian, Phys. Lett. 147B, 121 (1984). doi:10.1016/0370-2693(84)90605-1

[19] G. M. O'Brien and D. H. Tchrakian, Mod. Phys. Lett. A 4 (1989) 1389. doi:10.1142/S0217732389001581

[20] D. H. Tchrakian, Phys. Lett. 150B (1985) 360. doi:10.1016/0370-2693(85)90994-3 
[21] F. Navarro-Lerida, E. Radu and D. H. Tchrakian, Int. J. Mod. Phys. A 29 (2014) no.26, 1450149 doi:10.1142/S0217751X14501498 arXiv:1311.3950 [hep-th]].

[22] F. Navarro-Lerida and D. H. Tchrakian, Int. J. Mod. Phys. A 30 (2015) no.15, 1550079 doi:10.1142/S0217751X15500797 arXiv:1412.4654 [hep-th]].

[23] R. J. Szabo and O. Valdivia, JHEP 1406 (2014) 144 doi:10.1007/JHEP06(2014)144 arXiv:1404.4319 [hep-th]].

[24] V. N. Romanov, I. V. Frolov and A. S. Schwarz, Teor. Mat. Fiz. 37 (1978) 305. doi:10.1007/BF01018584

[25] Y. N. Obukhov and F. W. Hehl, Acta Phys. Polon. B 27 (1996) 2685 gr-qc/9602014. 